\title{
Color constancy in dermatoscopy with smartphone
}

\section{Blaž Cugmas, Franjo Pernuš, Boštjan Likar}

Blaž Cugmas, Franjo Pernuš, Boštjan Likar, "Color constancy in dermatoscopy with smartphone," Proc. SPIE 10592, Biophotonics-Riga 2017, 105920G (7 December 2017); doi: 10.1117/12.2297252

SPIE Event: The Second International Conference "Biophotonics-Riga 2017", 2017, Riga, Latvia 


\title{
Color constancy in dermatoscopy with smartphone
}

\author{
Blaž Cugmas*a,b, Franjo Pernuša $^{\mathrm{a}}$, Boštjan Likar ${ }^{\mathrm{a}}$ \\ ${ }^{a}$ Laboratory of Imaging Technologies, Faculty of Electrical Engineering, University of Ljubljana, \\ Tržaška 25, SI-1000 Ljubljana, Slovenia, EU \\ ${ }^{\mathrm{b}}$ Biophotonics laboratory, Institute of Atomic Physics and Spectroscopy, University of Latvia, \\ 19 Rainis Blvd., LV-1586, Riga, Latvia, EU
}

\begin{abstract}
The recent spread of cheap dermatoscopes for smartphones can empower patients to acquire images of skin lesions on their own and send them to dermatologists. Since images are acquired by different smartphone cameras under unique illumination conditions, the variability in colors is expected. Therefore, the mobile dermatoscopic systems should be calibrated in order to ensure the color constancy in skin images. In this study, we have tested a dermatoscope DermLite DL1 basic, attached to Samsung Galaxy S4 smartphone. Under the controlled conditions, jpeg images of standard color patches were acquired and a model between an unknown device-dependent RGB and a deviceindependent Lab color space has been built. Results showed that median and the best color error was 7.77 and 3.94, respectively. Results are in the range of a human eye detection capability (color error $\approx 4$ ) and video and printing industry standards (color error is expected to be between 5 and 6). It can be concluded that a calibrated smartphone dermatoscope can provide sufficient color constancy and can serve as an interesting opportunity to bring dermatologists closer to the patients.
\end{abstract}

Keywords: color constancy, color reproduction accuracy, dermatology, telemedicine, smartphone

\section{INTRODUCTION}

In the US, almost $5 \%$ of all new cancer cases appear on $\operatorname{skin}^{1}$. Melanoma is the most fatal skin cancer since it causes $74.2 \%$ of all skin cancer deaths. Despite its fatality, it can be successfully managed if discovered early ${ }^{2}$. Diagnostics is clinically done by dermatoscopy since dermatoscopes allow a noninvasive inspection of skin's superficial layer what offers an insight into pigmented lesions ${ }^{3}$. Therefore, dermatoscopy improves the diagnostic accuracy comparing to the naked eye clinicians ${ }^{2}$. Dermatoscope usually comprises magnifier and light source and it is designed for the manual use. Additionally, it can be mounted on the camera. High-class dermatoscopes can cost more than $1,000 €$, or even 2,000€ if a camera is included in the package. Therefore, just specialists (i.e. dermatologists) can economically and professionally justify the high purchase prices in their highly specialized practices.

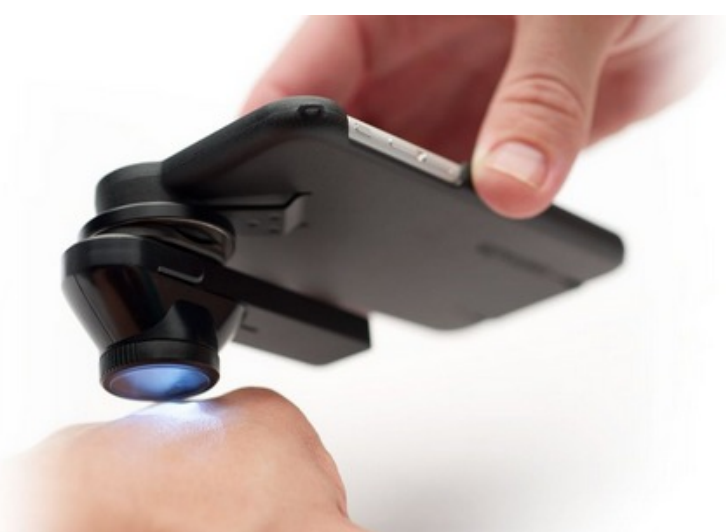

Figure 1. Smartphone with Dermlite ${ }^{\circledR}$ DL1 basic dermatoscope (from 3Gen, 2017 ${ }^{4}$ ).

*blaz.cugmas@fe.uni-lj.si; http://lit.fe.uni-lj.si/

Biophotonics-Riga 2017, edited by Janis Spigulis, Proc. of SPIE Vol. 10592

105920G · @ 2017 SPIE · CCC code: $1605-7422 / 17 / \$ 18$

doi: 10.1117/12.2297252

Proc. of SPIE Vol. 10592 105920G-1 
However, smaller and cheaper dermatoscopes for smart mobile phones have appeared lately; for example Dermlite ${ }^{\circledR}$ Hüd and DL1 basic (Figure 1) which cost $80 \$$ and $300 \$$, respectively. The aforementioned prices are affordable so these products are marketed for the patients. Since patients do not have the dermatological knowledge, they are encouraged to send skin lesion images to the specialists. These procedures already fall within the scope of telemedicine (also teledermatology) which offers relatively new modes of diagnosis, consultation and treatment with the help of telecommunications and information technology and has become accepted as a valid dermatological tool ${ }^{5}$.

In the suggested setting, dermatoscopic images will be acquired by different smartphone cameras under unique illumination conditions what can lead to various appearances of the same skin lesion. Especially, the color can be affected. Since melanomas are diagnosed with the help of ABCDE or 7-checker rules which include judgment of lesion color $^{6}$, it is crucial to calibrate images to obtain as realistic colors as possible. It was shown that color calibration method with professional dermatoscope and camera had ensured adequate color constancy ${ }^{7}$. However, it is questionable if the same results can be achieved with cheaper dermatoscope and camera. On the other hand, color constancy is crucial for prosperous teledermatology of skin lesions. In our study, we have built a system with affordable dermatoscope and average smartphone camera of limited photography options and the color constancy has been tested.

\section{MATERIAL AND METHODS}

Images were taken by a dermatoscopic system with a dermatoscope DermLite DL1 basic (3Gen, Inc., San Juan Capistrano, the USA). DermLite DL1 is an epiluminescence microscopy device, designed to be mounted on mobile device $^{4}$ and placed directly on the skin (Figure 1). Illumination is provided by 4 white LEDs which are separated from the skin by a spacer which enables cross-polarization in order to reduce reflection from the skin surface. It additionally includes a $15 \mathrm{~mm}$ lens. The dermatoscope is powered by a rechargeable battery which turns off automatically after approximately 2 minutes. Our measurements showed that the irradiance fluctuations were up to $0.9 \%$ during the operational period.

The dermatoscope was attached to the Samsung Galaxy S4 smartphone (Samsung Electronics Co. Ltd., Suwon, South Korea) with 13 MP camera $(\mathrm{f} / 2.2,31 \mathrm{~mm})$. Jpeg images were acquired by a program Open Camera (Mark Harman, gapps, Cambridge, the United Kingdom). Exposure time was not locked or it was locked to the brightest image (normally to patch no. 19 or 21), ISO/focus was set to auto, white balance to the daylight mode and all other camera/program features were turned off. When locked to white (no. 19) or to gray patch (no. 21), exposure time was $1 / 477$ and $1 / 167$ seconds, respectively.

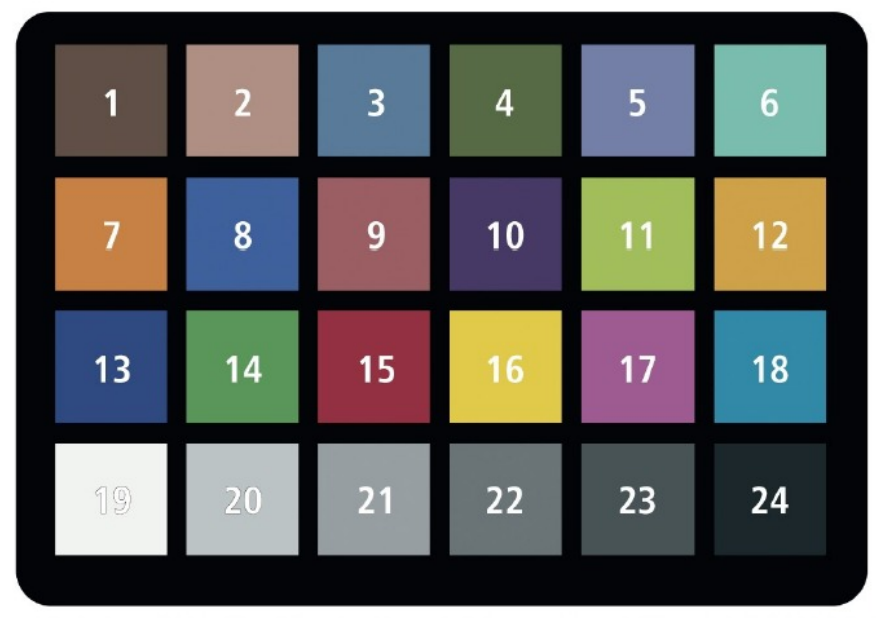

Figure 2. ColorChecker Classic (from X-rite, $2017^{8}$ ).

With the aforementioned dermatological system, images of ColorChecker Classic (X-rite, Michigan, the USA) were acquired (Figure 2). Considering acquisition, three sample groups were formed:

1. 24 images of ColorChecker, one per each color patch, were acquired. First, the exposure time was locked on the white color patch (no. 19, so called "neutral white") and after, the rest of patches were photographed. 
2. Patches no. 19 and 20 were discharged due to their high brightness comparing to other patches. The second acquisition had started on gray patch no. 21 ("neutral 6.5") and exposure time had been locked. The remaining patches were photographed in the descending order.

3. 24 images of 24 color patches were acquired without exposure time being locked. Before further processing, pixels of all images $\left(I_{c}\right)$ were normalized by the exposure time $e: I_{n}=I_{c} / e$.

The true color values (CIE L*a*b*) of patches were found in the Colorimetric data sheet ${ }^{8}$.

After the acquisition, a model between an unknown device-dependent RGB (sRGB) and a device-independent Lab color space (CIE L*a*b*) was built. First, correction of non-uniform illumination (i.e. shading correction) was done according to the method of Grana et $\mathrm{al}^{9}$. Briefly, for filter construction, only images of white, gray and black patches were considered (no. 19-24). For each image pixel $V_{C}(x, y)$ of color channel $C$, the filter $F_{C}(x, y)$ was built:

$$
F_{C}(x, y)=\frac{V_{C}(x, y)-R_{C}}{R_{C}},
$$

where $R_{C}$ is the most represented pixel value in a selected, well-illuminated area. After, we calculated $M_{C}(x, y)$ which represented a median of the measured values $F_{C}(x, y)$. Finally, original image pixel $I_{C}(x, y)$ is filtered:

$$
\widetilde{I}_{C}(x, y)=\frac{I_{C}(x, y)}{1+M_{C}(x, y)} .
$$

Color correction methods ${ }^{7,9}$ included additional procedures of image processing in order to deal with the nonlinearity of acquired images which happens due to the camera sensor's nonlinear response or visual enhancement procedures like gamma-curve. Since various polynomials for a relation estimation between true and real color values were used, we joined all procedures in just one function.

Images were then equally divided into training and test set. In order to avoid observer bias, random selection of sets was repeated, resulting in 100,000 unique combinations of training and test sets. For each combination, the difference between estimated and real colors was calculated.

Training acquisitions served for modeling a relation between recorded RGB $(R G B)$ and true CIE L*a*b* values $(L a b)$ which was based on polynomial functions:

$$
L a b=f(R G B) .
$$

All polynomial functions (Equation 3) are listed in Table 1. Polynomial coefficients were estimated by nonlinear regression, based on nlinfit function (Matlab R2015a, MathWorks Inc, the USA).

Table 1. Polynomial functions in nonlinear regression model (Equation 3).

\begin{tabular}{|l|l|}
\hline \multicolumn{1}{|c|}{ Number } & \multicolumn{1}{c|}{ Polynomial } \\
\hline 1 & $1 \mathrm{R} \mathrm{G} \mathrm{B}$ \\
\hline 2 & $\mathrm{R} \mathrm{G} \mathrm{B}$ \\
\hline 3 & $1 \mathrm{R} \mathrm{G} \mathrm{B} \mathrm{RG} \mathrm{RB} \mathrm{GB}$ \\
\hline 4 & $1 \mathrm{R} \mathrm{G} \mathrm{B} \mathrm{R}^{2} \mathrm{G}^{2} \mathrm{~B}^{2}$ \\
\hline
\end{tabular}

At the end, color error $(E)$ as a metric of color reproduction accuracy was calculated as an Euclidean distance between estimated $\left(L a b_{e s t}\right)$ and true $\left(L a b_{\text {true }}\right)$ test set colors:

$$
E=\sqrt{\left(\Delta L^{*}\right)^{2}+\left(\Delta a^{*}\right)^{2}+\left(\Delta b^{*}\right)^{2}} .
$$

Color errors (i.e. differences between two colors) below 4 are indistinguishable to human eyes, while the video and printing industry normally operates within the errors of 5 and $6^{7,10,11}$. 


\section{RESULTS}

Table 2 lists median and minimal color error of twelve models comprising three sample groups and four polynomials. Color calibration worked the best when polynomial "1RGB" was included, alone (polynomial 1) or with the extra coefficients (polynomials 3 and 4). With the sample group 2, median and minimal errors ran from 7.37 to 9.02 and from 3.94 to 4.85 , respectively. On average, that was up to $37 \%$ better compared to the situation when all patches are considered (group 1). The cause for the phenomenon could be found in Figure 3 where histograms of image mean RGB values are plotted. The most of the RGB values in group 1 images fall below 50 (out of 255) what appears very dark (if all three RGB components have the similar value). For example, patch no. 24 ("black") RGB values for illuminate D65 are $52^{8}$. So many low RGB values in a narrow range can easily lead to fitting difficulties because the signal is discrete and it can occupy only 256 values across the whole range. On the contrast, RGB values of group 2 images (exposure time was locked on patch no. 21) are more evenly distributed across the whole RGB range. Similar distribution can be seen in the group 3 (unlocked exposure time), however, there are few saturated acquisitions which have a considerable impact on the color calibration. A similar phenomenon was reported in the study by Grana et $\mathrm{al}^{9}$, where saturated acquisitions were not considered. Additional factors for bad model performance in the group 3 can be found in CCD characteristics and jpeg image enhancement ${ }^{7}$, however, the user has no influence over these factors on Galaxy S4 phone.

Table 2. Median and minimal (median / min) errors of models on all three sample groups when four polynomials are employed.

\begin{tabular}{|l|c|c|c|}
\hline $\begin{array}{c}\text { Sample group } \\
\text { Polynomial }\end{array}$ & $\begin{array}{c}\mathbf{2} \\
(24 / 24 \text { locked })\end{array}$ & $\begin{array}{c}\mathbf{3} \\
(22 / 24 \text { locked })\end{array}$ & $\begin{array}{c}\text { 3 } \\
(24 / 24 \text { unlocked })\end{array}$ \\
\hline $1(1 \mathrm{RGB})$ & $10.17 / 5.45$ & $7.37 / 4.83$ & $24.01 / 12.50$ \\
\hline $2(\mathrm{RGB})$ & $22.16 / 13.60$ & $14.40 / 8.32$ & $38.80 / 25.17$ \\
\hline $3(+\mathrm{RG} \mathrm{RB} \mathrm{GB})$ & $12.15 / 5.00$ & $7.77 / 3.94$ & $30.89 / 10.66$ \\
\hline $4\left(+\mathrm{R}^{2} \mathrm{G}^{2} \mathrm{~B}^{2}\right)$ & $10.78 / 5.69$ & $9.02 / 4.85$ & $36.42 / 13.42$ \\
\hline
\end{tabular}

Group 1

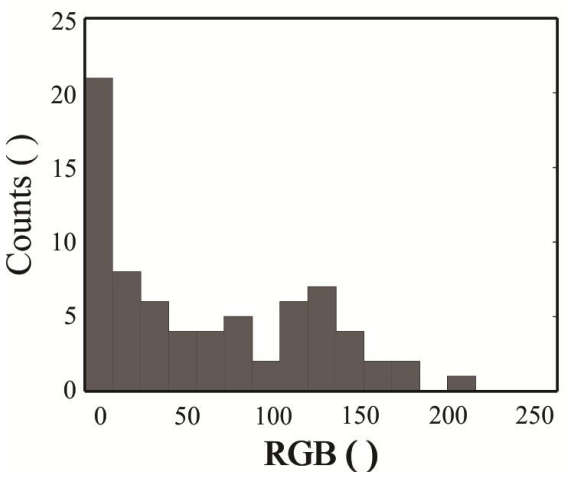

Group 2

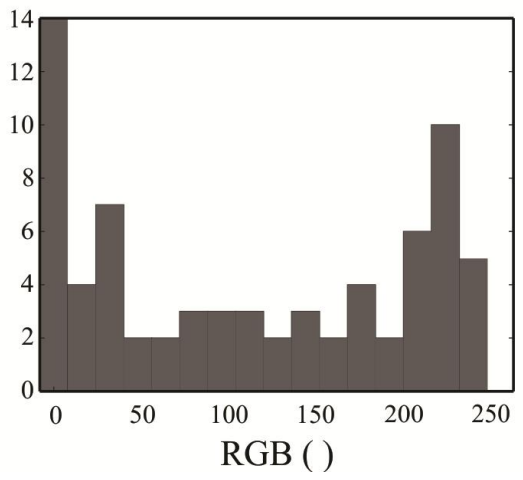

Group 3

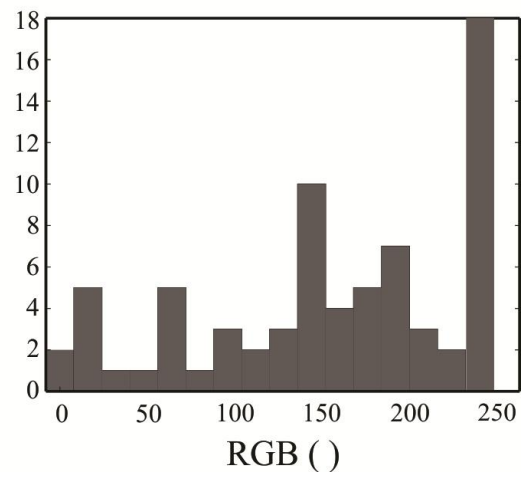

Figure 3. RGB value histograms of images for all three groups.

As mentioned before, a model with polynomial 1 (Table 1) performed satisfactorily. In agreement with the study of Quintana et $\mathrm{al}^{7}$, adding parameters to polynomial (polynomials 2, 3 and 4) does not decrease error median. On the other hand, the best result (i.e. minimal error) in all three groups was achieved when the polynomial 3 (1 R G B RG RB GB) was employed. Obviously, it is more suitable for modeling the nonlinear relation between RGB and Lab color spaces ${ }^{12}$.

It should be noted again that color errors below 4 are indistinguishable to the human eye, while the video in printing industry normally demands color error values to be around 5 or 6 . The best result (color error $=3.94$ ) was under 4 , so according to the literature, no difference in colors can be noticed (Figure 4). When it comes to the second model with the error value similar to the median (Table 2), slight differences are noticeable. Authors' eyes could spot a difference between purplish blue (patch no. 8, $5^{\text {th }}$ place in Figure 4) and gray patches (patch no. 23, $11^{\text {th }} /$ last place in Figure 4). 
Furthermore, when a color error is high $(=28.11$, Figure 4$)$, differences in estimated and true colors can be easily seen. However, there is not just a change in color shade, but a different basic color appeared. For example, blue flower patch (patch no. $5,4^{\text {th }}$ place in Figure 4 ) became orange to yellow. On the other hand, gray patches got more blue and violet shades.

Error: 3.94 Training: 125611131415161722 Test: 34789101218212324

True
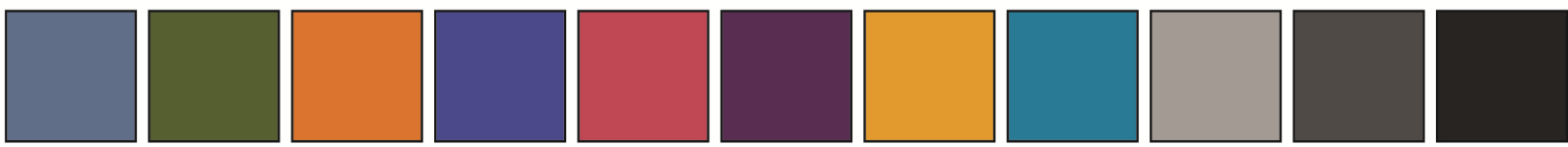

Est.
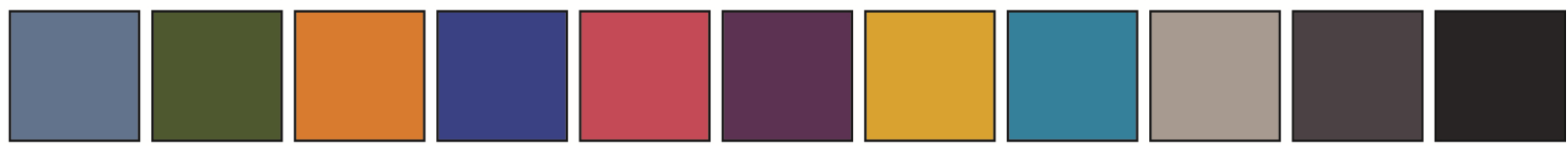

Error: 7.72 Training: 235910111214182124 Test: 14678131516172223

True
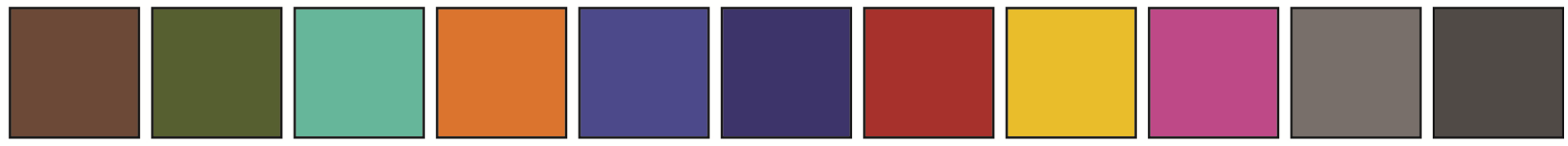

Est.
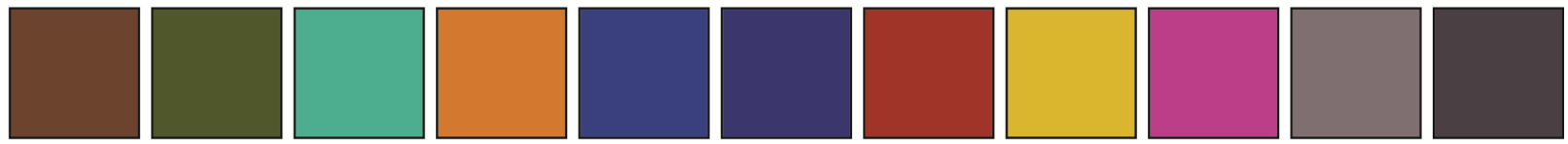

Error: 28.11 Training: 4781011131415161723 Test: 1235691218212224
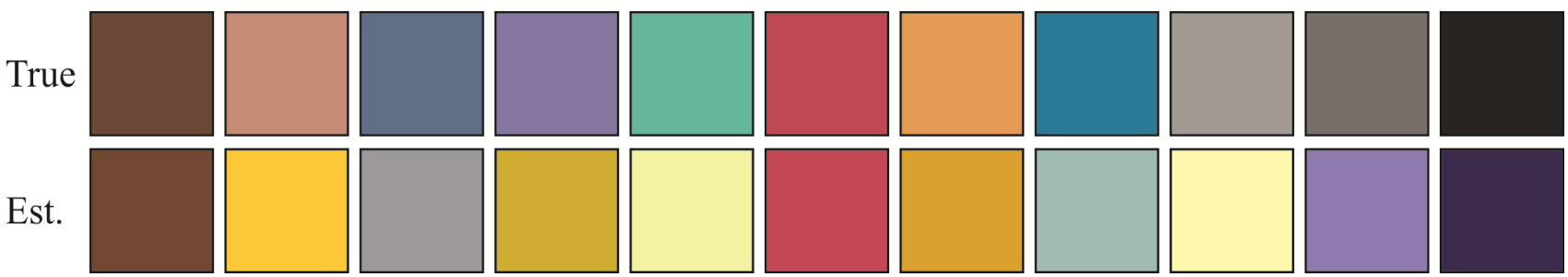

Figure 4. True and estimated (Est.) color patches of three models (test sets) with small, median and high color errors. Patch numbers in training and test sets are listed above color boxes.

\section{CONCLUSION}

Despite disregarding two brightest patches, a model from Figure 4 (polynomial 3, group 2) can be considered realistic since the skin is hardly as bright as patches 19 and 20. The best color error (i.e. the minimal differences between true and estimated color) is 3.94 what is indistinguishable to the human eye. Error median was 7.77 and as showed in Figure 4, color deviations from true values are hardly noticeable. What is more, more than $85 \%$ of all color patch combinations had an error less than 11. In our study, color patch combinations were randomly chosen what probably would not be the case in a realistic teledermatology application. There, color calibration would presumably not include the whole color range, but only colors which appear in the skin. We modestly speculate that having more realistic skin colors could even further improve color calibration procedure.

Our study showed that also a much cheaper dermatoscopic system with affordable dermatoscope and smartphone can achieve satisfactory color calibration results which are comparable to more expensive systems ${ }^{7}$. This is a good sign for the development of teledermatology since color-calibrated dermatoscope can involve patients in the diagnostic and therapeutic process and by that, raise patient health awareness. 


\section{ACKNOWLEDGMENTS}

The study was supported by Slovenian Research Agency (ARRS) (P2-0232, L2-5472, J2-5473, J7-6781, J2-7211, J27118) and by the Marie Skłodowska-Curie actions of the European Union (IF DogSPEC).

\section{REFERENCES}

[1] Siegel, R. L., Miller, K. D. and Jemal, A., "Cancer statistics, 2016,” CA. Cancer J. Clin. 66(1), 7-30 (2016).

[2] Rajpara, S. M., Botello, A. P., Townend, J. and Ormerod, A. D., "Systematic review of dermoscopy and digital dermoscopy/ artificial intelligence for the diagnosis of melanoma," Br. J. Dermatol. 161(3), 591-604 (2009).

[3] Kittler, H., Pehamberger, H., Wolff, K. and Binder, M., "Diagnostic accuracy of dermoscopy," Lancet Oncol. 3(3), 159-165 (2002).

[4] 3Gen., "DermLite DL1 basic," DermLite DL1 Basic, <https://dermlite.com/products/dermlite-dl1-basicsmartphone-skin-magnifier> (25 August 2017).

[5] Trettel, A., Eissing, L. and Augustin, M., "Telemedicine in dermatology: findings and experiences worldwide - a systematic literature review," J. Eur. Acad. Dermatol. Venereol., ahead of print (2017).

[6] Argenziano G, Fabbrocini G, Carli P, De Giorgi V, Sammarco E and Delfino M., "Epiluminescence microscopy for the diagnosis of doubtful melanocytic skin lesions: Comparison of the abcd rule of dermatoscopy and a new 7point checklist based on pattern analysis," Arch. Dermatol. 134(12), 1563-1570 (1998).

[7] Quintana, J., Garcia, R. and Neumann, L., "A novel method for color correction in epiluminescence microscopy," Comput. Med. Imaging Graph. 35(7), 646-652 (2011).

[8] X-rite, "Colorchecker Classic - Camera \& Image Calibration: X-Rite Photo \& Video.", $<$ http://xritephoto.com/colorchecker-classic $>$ (8 August 2017).

[9] C. Grana, G. Pellacani, S. Seidenari and R. Cucchiara., "Color calibration for a dermatological video camera system," Proc. 17th Int. Conf. Pattern Recognit. 3, 798-801 (2004).

[10] H. Brainard, D., "Color Appearance and Color Difference Specification," [The Science of Color], 191-216 (2003).

[11] Montserrat Corbalan-Fuertes, Maria Sagrario Millan Garcia-Verela and Maria Josefa Yzuel., "Color measurement in standard CIELAB coordinates using a 3CCD camera: correction for the influence of the light source," Opt. Eng. 39(6), 39-7 (2000).

[12] Lindbloom, B., "Useful Color Equations," Useful Color Equ., $<$ http://www.brucelindbloom.com/index.html?Eqn_RGB_XYZ_Matrix.html> (24 August 2017). 